\title{
DISCOVERY OF A PROBABLE GRAVITATIONAL LENS
}

\author{
G. Meylan \\ European Southern Observatory \\ D-8046 Garching bei München, FRG. \\ S. Djorgovski \\ Palomar Observatory, California Institute of Technology \\ Pasadena, CA 91125, USA.
}

\begin{abstract}
We report the discovery of a new probable gravitational lens system, associated with the quasar UM $425=1120+019$, at $z=1.465$. The optical image of the system consists of 4 or more components. The two wellobserved brightest components have very similar spectra. The difference of the two spectra (scaled appropriately) is consistent with a spectrum of a galaxy at $z \simeq 0.6$. There is a large number of faint galaxies in the field, suggestive of a lensing cluster at that redshift.
\end{abstract}

The quasar UM 425 has been selected for an optical imaging search for gravitational lenses, due to its moderately high redshift $(z \simeq 1.49)$ and the apparently large luminosity, both of which make it a good a priori case for gravito-optical magnification. The survey is described in more detail in the companion paper by Djorgovski \& Meylan.

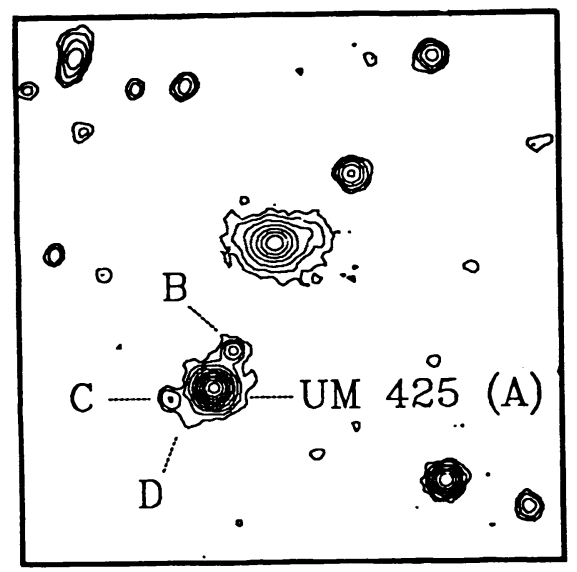

Figure 1: A section of a $V+R$ stack image of the UM 425 field, obtained at CTIO. The field shown is $50 \times 50$ arcsec, with north to the top, east to the left. The components of the UM 425 system are indicated with the letters. The isophotal levels are spaced logarithmically in factors of 2 .

Multicolor CCD images of the object, obtained at CTIO in March of 1987 (Figure 1) show several close companions (within 3 to 6 arcsec), whose $B V R$ colors 271

D. E. Osterbrock and J. S. Miller (eds.), Active Galactic Nuclei, 271-272.

(C) 1989 by the IAU. 
appear to be similar or identical to those of the principal quasar image (UM 425 A) itself. There appears to be a moderately distant foreground cluster in the field. The apparent magnitude of the component $\mathrm{A}$ is $V \simeq 16.2$; the components $\mathrm{B}$ and $\mathrm{C}$ are $\sim 4.6^{m}$ and $5.6^{m}$ dimmer in $V$ respectively. The component B has the same $B V R$ colors as the component $\mathrm{A}$, within the measurement errors; the colors of $\mathrm{C}$ are slightly different. Both components $\mathrm{C}$ and $\mathrm{D}$ appear slightly non-stellar. The brightest component, A, appears unresolved on our CCD images. R. Perley kindly obtained a brief VLA snapshot of the field, and established that the system is not brighter than about a third of a mJy at 6 and $20 \mathrm{~cm}$.

The long-slit spectra obtained at ESO, Palomar, and Las Campanas in April and May of 1988 , indicate that the faint components B, and possibly also C, have essentially the same spectra (Figure 2) as the brightest image (A). The redshift of the quasar, based on the "clean" C III] 1909 line is $z=1.465 \pm 0.005$. The crosscorrelation redshift difference between the components $\mathrm{A}$ and $\mathrm{B}$ is consistent with zero, with $\sim 200 \mathrm{~km} / \mathrm{s}$ uncertainty.

Prompted by the slightly redder color of the component $B$, we scaled the spectrum of the component A by 0.01 , and subtracted it from the spectrum of the component $\mathrm{B}$. The residual can be interpreted as a spectrum of a $R \simeq 22.5 \mathrm{mag}$ galaxy at $z \simeq 0.6$, corroborating the gravitational lens interpretation.

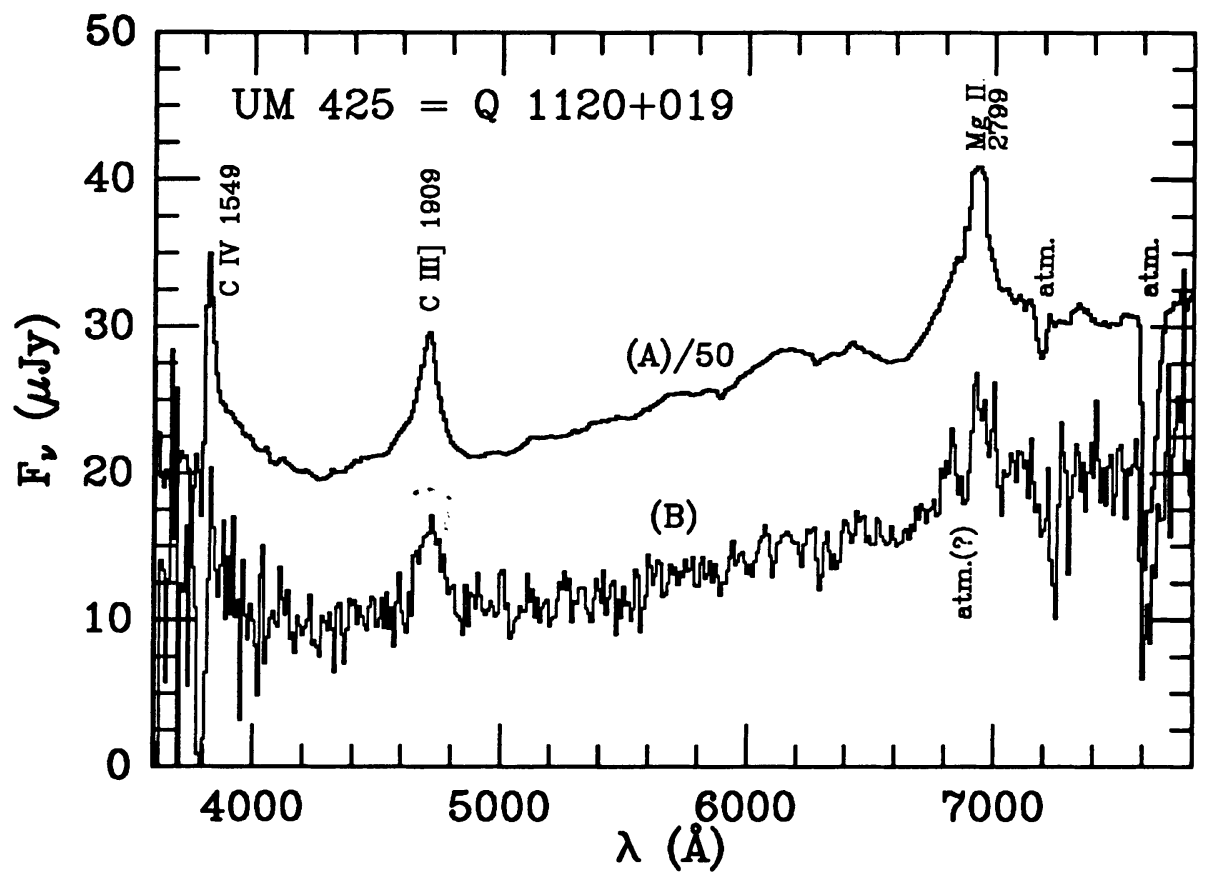

Figure 2: CCD spectra of components A and B, obtained at ESO. The brighter component (A) was scaled down by a factor of 50 , for an easier comparison. There may be some associated absorption in the Mg II 2799 and C IV 1549 lines, but the presence of the atmospheric B-band and the noise shortward of $\sim 3800 \AA$ make the interpretation difficult. 